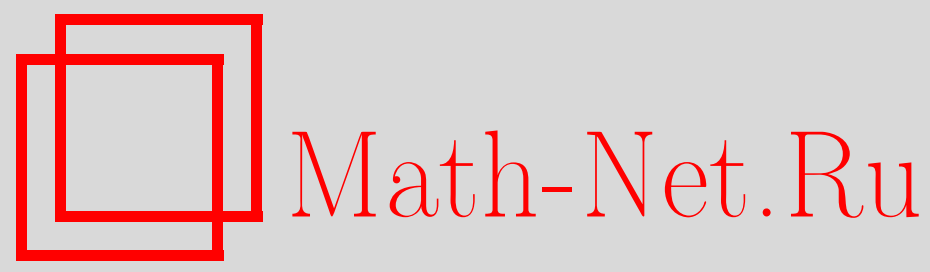

Л. Г. Куракин, Критические случаи устойчивости. Обращение теоремы о неявной функции для динамических систем с косимметрией, Матем. заметки, 1998, том 63, выпуск $4,572-578$

DOI: https://doi.org/10.4213/mzm1317

Использование Общероссийского математического портала Math-Net.Ru подразумевает, что вы прочитали и согласны с пользовательским соглашением http://www . mathnet.ru/rus/agreement

Параметры загрузки:

IP : 52.205 .19 .152

26 апреля 2023 г., $16: 19: 41$

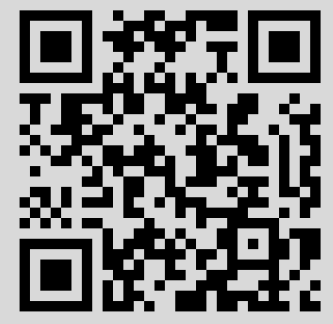




\title{
КРИТИЧЕСКИЕ СЛУЧАИ УСТОЙЧИВОСТИ. ОБРАЩЕНИЕ ТЕОРЕМЫ О НЕЯВНОЙ ФУНКЦИИ ДЛЯ ДИНАМИЧЕСКИХ СИСТЕМ С КОСИММЕТРИЕЙ
}

\author{
Л. Г. Куракин
}

В работе получены критерии устойчивости граничных равновесий для трех критических случаев: $(n, k)=(3,0),(2,1),(1,1)$.

Библиограффия: 16 названий.

Понятие косимметрии векторного поля в гильбертовом пространстве или на римановом многообразии введено в работе [1] - векторное поле, ортогональное в каждой точке данному. Более общее определение [2]: косимметрия - дифференциальная 1-форма, аннулируюшая данное векторное поле в каждой точке.

Векторные поля, далее, рассматриваются на конечномерном многообразии, а все рассуждения носят локальный характер. Поэтому ограничимся рассмотрением векторных полей на $\mathbb{R}^{n}$, используя обычные для линейного пространства отождествления точек с векторами, векторных полей с отображениями и т.д.

Непрерывное однопараметрическое семейство равновесий является обычньм объектом для векторных полей с нетривиальной косимметрией [1], [3]-[5]. Спектр устойчивости равновесия меняется вдоль такого семейства [3], но всегда содержит точку нуль. Справедлив принцип линеаризации [6]: равновесие упомянутого семейства устойчиво, если весь его спектр устойчивости, кромепростого нулевого собственного значения, лежит строго в левой полуплоскости (следует из общих результатов А. М. Ляпунова для критического случая устойчивости при простом нулевом корне), и неустойчиво, если этот спектр содержит точку из правой полуплоскости.

Устойчивость равновесия, здесь и далее, понимается как нейтральная устойчивость вдоль семейства равновесий и, одновременно, асимптотическая устойчивость в трансверсальных к нему направлениях.

Семейство равновесий в условиях общего положения разбивается на устойчивые и неустойчивые по линейному приближению дуги. Разделяющие их равновесия будем называть граничными. Соседними могут оказаться не только устойчивая дуга с неустойчивой, но и две устойчивые или две неустойчивые дуги. Спектр устойчивости граничного равновесия расположен в замыкании левой полуплоскости, и его нейтральная часть,

Работа выполнена при поддержке Российского фонда фундаментальных исследований, грант № 96-01-01791, и Международного научного фонда, грант NRQ000. 
лежащая на мнимой оси, отлична от простого нулевого собственного значения. В задаче устойчивости такого равновесия имеет место критический случай, т.е. линейного приближения недостаточно для ее решения.

В данной работе приведены критерии устойчивости граничного равновесия во всех критических случаях коразмерности 0 и 1 (см. замечание 1 ), кроме следующих двух случаев нейтрального спектра устойчивости:

1) двукратное нулевое собственное значение с индексом 2 ;

2) простые нуль и две пары чисто мнимых собственных значений.

Первьй случай нет необходимости рассматривать, поскольку он полностью исследован Ляпуновым [7]. Что касается второго, то он непрост, но достаточно полное решение задачи устойчивости для него можно извлечь из результатов работы [8].

Наличие косимметрии определяет специфику рассматриваемых вырождений. В определенном смысле верно и обратное. Пусть динамическая система допускает гладкое однопараметрическое семейство равновесий. Тогда по крайней мере локально вблизи каждого невырожденного равновесия, входящего в состав семейства, можно определить нетривиальную косимметрию такую, что равновесия семейства оказьваются некосимметричными, т.е. не являются равновесиями самой косимметрии.

\section{1. Обращение теоремы о неявной функции для косимметрических сис-} тем. Рассмотрим дифференциальное уравнение

$$
\dot{u}=F(u) \text {. }
$$

Векторное поле $F: \Omega \rightarrow \mathbb{R}^{n}$ является аналитическим в некоторой области $\Omega \subset \mathbb{R}^{n}$, имеет равновесие $u_{0} \in \Omega$ и допускает непрерьвную косимметрию $L: \Omega \rightarrow \mathbb{R}^{n}$. Последнее означает [1], [3], что в каждой точке $u \in \Omega$ векторы $F(u)$ и $L(u)$ ортогональны: $(F(u), L(u))=0$.

Считаем вьполненньми следующие предположения:

i) равновесие $u_{0}$ некосимметрично: $L\left(u_{0}\right) \neq 0$;

ii) размерность $\operatorname{dim} \operatorname{ker} A_{0}=1$, где оператор $A_{0}=F^{\prime}\left(u_{0}\right)$.

Тем самым, выполнены условия теоремы о неявной функции для косимметрических уравнений [1], [3]-[5], из которой следует, что уравнение (1) имеет однопараметрическое семейство $S_{1}$ некосимметричных равновесий, включающее в себя равновесие $u_{0}$ и задаваемое аналитическим отображением $T_{1}: J_{1} \rightarrow \Omega$, так что $S_{1}=T_{1}\left(J_{1}\right)$. Здесь $J_{1}=$ $\operatorname{ker} A_{0} \cap \Omega_{1}$, где $\Omega_{1} \subset \Omega$ - некоторая окрестность точки $u_{0}$. В окрестности $\Omega_{1}$ нет равновесий, не принадлежащих указанному семейству.

Возникает вопрос о существовании нетривиальной косимметрии у динамической системы, обладающей непрерьвньм однопараметрическим семейством равновесий. Мы же ограничимся тем результатом, который необходим в дальнейшем.

Рассмотрим в пространстве $\mathbb{R}^{n}$ автономное дифференциальное уравнение с нулевым равновесием

$$
\dot{u}=Q(u), \quad Q(0)=0 .
$$

Векторное поле $Q: \mathfrak{D} \rightarrow \mathbb{R}^{n}$ является аналитическим в некоторой окрестности нуля $\mathfrak{D} \subset \mathbb{R}^{n}$.

Следующую теорему можно рассматривать как обращение теоремы о неявной функции для косимметрических уравнений. Пусть $X_{1}$ - ортогональное дополнение к $X_{0}$, 
$J=X_{0} \cap \mathfrak{D}, J^{\prime}$ - некоторый, меньший $J$, отрезок прямой $X_{0}$, содержащий нуль строго внутри себя.

ТЕорема 1. Пусть выполнены следующие условия:

1) точка $\lambda_{0}=0$ принадлежит спектру $\sigma(A)$ оператора $A=Q^{\prime}(0)$, причем этому собственному значению отвечает одна жсорданова клетка, т.е. $\operatorname{dim} X_{0}=1$, әде $X_{0}=\operatorname{ker} A$

2) векторное поле $Q$ имеет однопараметрическое семейство $S$ равновесий, параметризуемое отображением

$$
V: J^{\prime} \rightarrow \mathfrak{D}, \quad V: u \mapsto u+T(u)
$$

где $T: J^{\prime} \rightarrow X_{1}$ - аналитическое отображение, которое обращается в 0 в точке $u=0$ вместе со своей производной: $T(0)=T^{\prime}(0)=0$.

Тогда в некоторой окрестности нуля $\mathfrak{D}_{1} \subset \mathfrak{D}$ можно задать аналитическую косимметрию $\mathfrak{L}$ уравнения (2) такую, что равновесия семейства $S$ некосимметричны: $\mathfrak{L}\left(u_{*}\right) \neq 0$ в каждой точке $u_{*} \in S \cap \mathfrak{D}_{1}$.

ДоКАЗАТЕЛЬСТвО. Пусть $P_{0}$ - ортогональный проектор на подпространство $X_{0}$. Ему соответствует дополнительньй проектор $P_{1}=I-P_{0}$ на подпространство $X_{1}=\operatorname{im} P_{1}$.

Замена переменной

$$
v=\Phi(u)=u-T\left(P_{0}(u)\right)
$$

выпрямляет множество $S$. Это означает, что семейство равновесий преобразованного уравнения (2)

$$
\dot{v}=G(v), \quad G(0)=0
$$

целиком заполняет окрестность нуля прямой $P_{1} v=0$, т.е. равенство $G\left(P_{0} v\right)=0$ выполняется для каждой точки $v \in \mathfrak{D}$. Следовательно, векторное поле $G$ представимо в виде $G: v \mapsto G(v)=K(v) P_{1} v$, где $K(v)$ - (определяемая неоднозначно) линейная оператор-функция, гладко зависящая от вектора $v$, причем $K(0)=G^{\prime}(0)=A$.

Поля $Q$ и $G$ связаны соотношением $Q=\left[\Phi^{\prime}\right]^{-1} G \Phi$. Для доказательства теоремы 1 достаточно найти нетривиальную косимметрию $L$ уравнения $(4)$, поскольку поле $\mathfrak{L}=$ $\left[\Phi^{\prime *}\right]^{-1} L \Phi$ будет косимметрией поля $Q$, и $\mathfrak{L}(0) \neq 0$, если $L(0) \neq 0$.

Из тождества $(G(v), L(v))=\left(P_{1} v, K^{*}(v) L(v)\right)$, в котором звездочка соответствует сопряженному оператору, следует, что всякий оператор $L$, удовлетворяющий равенству

$$
K^{*}(v) L(v)=0
$$

есть косимметрия оператора $G$.

Имеют место разложения $K^{*}(v)=K_{0}^{*}(v)+K_{1}^{*}(v), L(v)=L_{0}(v)+L_{1}(v)$, где оператор-функции $K_{i}(v)$ и операторы $L_{i}$ определяются следующим образом: $K_{i}(v)=P_{i} K(v)$, $L_{i}=P_{i} L(i=0,1)$. Справедливы тождества $K_{0}^{*}(v) L_{1}(v)=K^{*}(v) P_{0}^{*} P_{1} L(v)=0$ (учли, что $\left.P_{0}^{*}=P_{0}\right), K_{1}^{*}(v) L_{0}(v)=0$. Это позволяет записать уравнение $(5)$ в виде

$$
K_{0}^{*}(v) L_{0}(v)+K_{1}^{*}(v) L_{1}(v)=0 .
$$


Нетривиальной косимметрией уравнения (4) является, например, векторноеполе $L: v \mapsto$ $L(v)=e_{0}+L_{1}(v)$, где $e_{0} \in X_{0}$ - произвольньй фиксированньй ненулевой вектор, а отображение $L_{1}$ определяется из уравнения (6):

$$
L_{1}: v \mapsto L_{1}(v)=-\left[K_{1}^{*}(v)\right]^{-1} K_{0}^{*}(v) e_{0} .
$$

Так как оператор $K_{1}^{*}(0)=P_{1} A^{*}$ обратим, оператор $\left[K_{1}^{*}(v)\right]^{-1}$ существует и аналитичен для всех $v$ из некоторой окрестности нуля $\mathfrak{D}_{2} \subset \mathfrak{D}$.

СлЕДСТВИЕ. Пусть уравнение (1) с аналитической правой частью F обладает всеми указанными выше свойствами: допускает непрерывную косимметрию $L$, имеет равновесие $u_{0}$. Пусть выполнены предположсения і) $u$ iі). Тогда в некоторой окрестности точки $u_{0}$ определена и аналитическая нетривиальная косимметрия $\mathfrak{L}$ этого уравнения, причем $\mathfrak{L}\left(u_{0}\right) \neq 0$.

2. Критические случаи устойчивости. Будем рассматривать только те вырождения задачи устойчивости равновесия $u_{0}$ косимметрического уравнения $(1)$, при которых не нарушаются условия і) и іi) теоремы о неявной функции для динамических систем с косимметрией, что гарантирует существование семейства $S_{1}$ равновесий. Теорема 1 позволяет в разобранных далее критических случаях устойчивости косимметрическое уравнение (1) рассматривать просто как уравнение, имеющее семейство равновесий, не накладывая каких-либо дополнительных ограничений (т.е. как уравнение (2) в условиях 1) и 2) теоремы 1). Считая, что семейство $S$ равновесий локально расположено на прямой, чего всегда можно добиться заменой $(3)$, приходим к задаче устойчивости нулевого равновесия уравнения (4).

Предположим, что весь спектр $\sigma(A)$ лежит на мнимой оси. Согласно принципу сведения [9], к такому случаю редуцируется и общая задача устойчивости равновесия, когда спектр $\sigma(A)$ лежит в замыкании левой полуплоскости (это выполнено для граничных равновесий).

Рассмотрим три различных случая нейтрального спектра устойчивости.

1. Трехкратное нулевое собственное значение: $\sigma(A)=\{0\}$. Уравнение (4) трехмерно, в жордановом базисе матрицы $A$ имеет следующую координатную форму записи:

$$
\dot{x}_{1}=x_{2}+g_{1}(x), \quad \dot{x}_{2}=x_{3}+g_{2}(x), \quad x_{3}=g_{3}(x) \text {. }
$$

Здесь $x=\left(x_{1}, x_{2}, x_{3}\right) \in \mathbb{R}^{3}, g_{i}: \mathfrak{D} \rightarrow \mathbb{R}$ - аналитические функции $(i=1,2,3)$, разложения которых в ряд Тейлора начинаются со слагаемых не ниже второй степени, причем $g_{i}\left(x_{1}, 0,0\right)=0(i=1,2,3)$ для всех векторов $\left(x_{1}, 0,0\right) \in \mathfrak{D} \subset \mathbb{R}^{3}$.

ТЕОрема 2. Нулевое равновесие системы (7) неустойчиво, если величина $a \neq 0$, əде $a=\frac{\partial^{2} g_{3}}{\partial x_{1} \partial x_{2}}(0)$.

ДокАЗАТЕЛЬство. Квазиоднородная система

$$
\dot{x}_{1}=x_{2}, \quad \dot{x}_{2}=x_{3}, \quad \dot{x}_{3}=a x_{1} x_{2}
$$

имеет растушие решения вида инвариантный луч [10]:

$$
x_{1}=k r^{2}, \quad x_{2}=2 k r^{3}, \quad x_{3}=6 k r^{4}, \quad \dot{r}=r^{2}, \quad k=12 / a .
$$


Далее проходят стандартные рассуждения [10]. Введем обобщенные сферические координаты

$$
x_{1}=k R^{2}, \quad x_{2}=2 k R^{3}\left(1+\theta_{1}\right), \quad x_{3}=6 k R^{4}\left(1+\theta_{2}\right),
$$

где $R \geqslant 0, \theta_{i} \in \mathbb{R}(i=0,1)$. Когда переменная $R \rightarrow 0$, а $\theta=\left(\theta_{1}, \theta_{2}\right)$ меняется внутри некоторой окрестности нуля $W \subset \mathbb{R}^{2}$, система (7) имеет в этих координатах следующую асимптотику:

$$
\dot{R}=R^{2}\left(1+\theta_{1}\right)+o\left(R^{2}\right), \quad \dot{\theta}=R f(\theta)+o(R) .
$$

Здесь $f=\left(f_{1}, f_{2}\right)$ - вектор-функция:

$$
f_{1}(\theta)=-3\left(1+\theta_{1}\right)^{2}+3\left(1+\theta_{2}\right), \quad f_{2}(\theta)=-4\left(1+\theta_{1}\right) \theta_{2} .
$$

Рассмотрим двумерную систему $\dot{\theta}=f(\theta)$. Ее равновесие $\theta=0$, отвечающее растущему лучу (8), асимптотически устойчиво в линейном приближении. Достаточное условие неустойчивости нулевого равновесия системы (7) выполнено (см. [10, с. 86]).

2. Двукратное нулевое и простая пара чисто мнимых собственных значений: $\sigma(A)=$ $\{0, \pm i \omega\}, \omega>0$. Перейдем к комплексной координатной форме записи уравнения (4) в жордановом базисе матрицы $A$, раскладьвая правую часть $G$ в ряд Тейлора. Затем надлежащей заменой переменных удалим нерезонансные квадратичные слагаемые [11]. Получим систему

$$
\begin{gathered}
\dot{x}_{1}=x_{2}+a x_{1} x_{2}+a_{1} x_{2}^{2}+a_{2}|z|^{2}+q_{1}(y), \quad \dot{x}_{2}=b x_{1} x_{2}+b_{1} x_{2}^{2}+b_{2}|z|^{2}+q_{2}(y), \\
\dot{z}=i \omega z+C_{1} x_{1} z+C_{2} x_{2} z+q_{3}(y) .
\end{gathered}
$$

Здесь $y=\left(x_{1}, x_{2}, z, \bar{z}\right), x_{1}, x_{2} \in \mathbb{R}, z \in \mathbb{C}$; аналитические функции $q_{i}: W \rightarrow \mathbb{R}(i=1,2)$, $q_{3}: W \rightarrow \mathbb{C}$ определены в некоторой окрестности нуля $W \subset \mathbb{R}^{2} \times \mathbb{C}^{2}$, отвечающей области $\mathfrak{D}$, причем $\left|q_{j}(y)\right|=o\left(\|y\|^{2}\right)$ при $\|y\| \rightarrow 0(j=1,2,3)$. Коэффициенты $a, b, a_{i}, b_{i}$ вещественны, а $C_{i}$ комплексны $(i=1,2)$.

Теорема 3. Нулевое равновесие системы (9) неустойчиво, если коэффициент $b \neq 0$.

ДокАЗАТЕльСтво. Введем новые координаты $R, \theta_{1}, \theta_{2}, \varphi \in \mathbb{R}$, полагая

$$
x_{1}=l R, \quad x_{2}=l R^{2}\left(1+\theta_{1}\right), \quad z=R^{2} \theta_{2} \exp (i \varphi), \quad l=2 / b, \quad R, \theta_{2}>0 .
$$

Переписанная в этих координатах система (9) имеет следующее асимптотическое представление:

$$
\begin{gathered}
\dot{R}=R^{2} h(\theta)+o\left(R^{2}\right), \quad \dot{\theta}=R \psi(\theta)+o(R), \quad \dot{\varphi}=\Phi(R, \theta, \varphi), \\
h=1+\theta_{1}, \quad \psi=\left(\psi_{1}, \psi_{2}\right), \quad \psi_{1}=-2 h \theta_{1}, \quad \psi_{2}=\left(-2 h+l \operatorname{Re} C_{1}\right) \theta_{2},
\end{gathered}
$$

когда $R \rightarrow 0$, а переменная $\theta=\left(\theta_{1}, \theta_{2}\right)$ принадлежит области $W_{\delta}=\left\{\theta \in \mathbb{R}^{2}:\left|\theta_{1}\right|<\delta\right.$, $\left.0<\theta_{2}<\delta\right\}, \delta>0$. Явньй вид известной функции $\Phi$ не имеет значения для дальнейших рассуждений.

Обратимся теперь к системе $\dot{\theta}=\psi(\theta)$. Она имеет равновесие $\theta_{0}=0$ такое, что вьполнено неравенство $h\left(\theta_{0}\right)>0$. Матрица $\Gamma=\psi^{\prime}(0)$ имеет собственные значения $\lambda_{1}=-2$, 
$\lambda_{2}=l \operatorname{Re} C_{1}-2$. Нулевое равновесие системы (9) неустойчиво при любом значении $\lambda_{2} \in \mathbb{R}$. Это следует из общей теоремы о неустойчивости (см. [10, с. 86]), если равновесие $\theta_{0}$ асимптотически устойчиво в линейном приближении $\left(\lambda_{2}<0\right)$. В случае $\lambda_{2} \geqslant 0$ соответствуюшие функции Четаева построены в работе $[12$, c. 18$]$ при изучении проблемы в обшей форме.

3. Простые нуль и пара чисто мнимых собственных значений: $\sigma(A)=\{0, \pm i \omega\}$, $\omega>0$. Трехмерное уравнение (4), нормализованное до слагаемых четвертого порядка включительно [11], имеет следующую асимптотику при $|(x, z, \bar{z})| \rightarrow 0$ :

$$
\begin{gathered}
\dot{x}=b_{0}|z|^{2}+b_{1} x|z|^{2}+b_{2} x^{2}|z|^{2}+b_{3}|z|^{4}+o\left(x^{3}|z|+|z|^{4}\right), \\
\dot{z}=i \omega z+D_{1} x z+D_{2} x^{2} z+D_{0} z|z|^{2}+o\left(x^{2}|z|+|z|^{3}\right) .
\end{gathered}
$$

Здесь $x$ - вещественная, а $z$-комплексная переменные; коэффициенты $b_{l}(l=0,1,2,3)$ вешественны, а $D_{j}=d_{j}+i \delta_{j}$ комплексны, $d_{j}, \delta_{j} \in \mathbb{R}(j=0,1,2)$.

Будем говорить, что нулевое равновесие системы (10) $z$-устойчиво, если оно устойчиво по Ляпунову [6] и, одновременно, асимптотически устойчиво по отношению к переменной $z$ [7], [13].

Теорема 4. Справедливь следующие утверждения об устойчивости нулевого равновесия системы (10):

1) равновесие $z$-устойчиво, если $b_{0} d_{1}<0$, и неустойчиво, если $b_{0} d_{1}>0$;

2) в вырохденном случае $d_{1}=0, b_{0} \neq 0$ равновесие $z$-устойчиво, если $d_{2}<0$, и неустойчиво, если $d_{2}>0$;

3) в вырожденном случае $b_{0}=0, d_{1} \neq 0$ равновесие $z$-устойчиво при выполнении любого из следующих условий:

a) $k_{1}<0, k_{2}<0$,

б) $k_{1}>0, k_{3}<0$,

и неустойчиво, если $k_{2}>0$ или справедливы неравенства $k_{1}>0, k_{3} \geqslant 0$. Здесь $k_{1}=2 d_{0}+b_{1}, k_{2}=2 b_{3} d_{1}-2 d_{0} b_{1}, k_{3}=k_{1}^{2}+4 k_{2}$.

Для доказательства $z$-устойчивости нулевого равновесия системы (10) в каждом случае строится определенно-положительная функция, производная которой в силу этой системы является определенно-отрицательной по отношению к переменной $z$ [13]. За единственным исключением, ее удается построить в форме полинома. Труднее оказался случай 3б), в котором строится трансцендентная функция Ляпунова и доказывается, что полиномиальных не существует. Подробнее это будет изложено в другой работе.

Для обоснования условий неустойчивости достаточно проверить вьполнение общих достаточных условий неустойчивости, полученных в работах [14], [15].

ЗАмечАниЕ 1 . Случай 1$)$ теоремы $4\left(b_{0} d_{1} \neq 0\right)$ встречается как случай общего положения в уравнениях с косимметрией. Случаи вырождения задачи устойчивости граничного равновесия, рассмотренные в теоремах 2-4, имеют коразмерность 1.

ЗАМЕчАнИЕ 2 . Неустойчивость нулевого равновесия системы (10) в случае $b_{0} d_{1}>0$ следует из результатов работы [16].

Автор благодарит В.И. Юдовича за полезные обсуждения. 


\section{СПИСОК ЦИТИРОВАННОЙ ЛИТЕРАТУРЫ}

[1] Юдович В.И.Косимметрия, вырождение решений операторных уравнений, возникновение фильтрационной конвекции // Матем. заметки. 1991. Т. 49. № 5. С. 142-148.

[2] Юдович В.И. Косимметрия и дифференциальные уравнения второго порядка // Деп. ВИНИТИ № 1008-В93. М.: ВИНИТИ, 1993.

[3] Yudovich V.I. Secondary cycle of equilibria in a system with cosymmetry, its creation by bifurcation and impossibility of symmetric treatment of it // Chaos. 1995. V. 2. № 5. P. 402-411.

[4] Юдович В.И. Теорема о неявной функции для косимметрических уравнений // Матем. заметки. 1996. Т. 60. №2. С. 313-317.

[5] Yudovich V. I. The cosymmetric version of the implicit function theorem / Seminar "Linear Topological Spaces and Complex Analysis". II / ed. A. Aytuna. Ankara: Middle East Technical Univ., 1995. P. 105-125.

[6] Ллпунов А. М. Общая задача об устойчивости движения. М.: Гостехиздат, 1950.

[7] Ллпунов А. М. Исследование одного из особенных случаев задачи об устойчивости движения // Собр. сочинений. Т. 2. М.: АН СССР, 1956. С. 272-331.

[8] Хазин Л. Г. Условия устойчивости равновесия при двух парах чисто мнимых и одном нулевом корне. Препринт № 50. М.: ИПМ АН СССР, 1986.

[9] Плисс В.А.Принцип сведения в теории устойчивости движения // Изв. АН СССР. Сер. матем. 1964. Т. 28. №6. С. 1297-1324.

[10] Хазин Л.Г., Шноль Э.Э. Устойчивость критических положений равновесия. Пущино: ОНТИ НЦБИ АН СССР, 1985.

[11] Арнольд В. И. Дополнительные главы теории обыкновенных дифференциальных уравнений. М.: Наука, 1978.

[12] Шноль Э.Э., Хазин Л. Г. Об устойчивости стационарных решений общих систем дифференциальных уравнений вблизи критических случаев. Препринт №91. М.: ИПМ АН СССР, 1979.

[13] Румянцев В.В.Об устойчивости движения по отношению к части переменных // Вестн. МГУ. Сер. 1. Матем., мех. 1957. № 4. С. 9-16.

[14] Каменков Г. В. Избранные труды. Т. 2. Устойчивость и колебания нелинейных систем. М.: Наука, 1972.

[15] Малкин И.Г. Теория устойчивости движения. М.: Наука, 1966.

[16] Хазин Л.Г. Об устойчивости положений равновесия в некоторых критических случаях. Препринт №10. М.: ИПМ АН СССР, 1979.

Ростовский государственный университет

Поступило

E-mail: kurakin@mmf.unird.ac.ru 06.08 .96 